\title{
The effect of vacuum polarization on the propagation of charged particles and light
}

\author{
O efeito da polarização do vácuo na propagação \\ de particulas carregadas e luz
}

Simon Davis ${ }^{1}$

\begin{abstract}
An examination of the physical phenomena of vacuum polarization provides support for the effect of the properties of the medium on the propagation of charged particles and light. The implications for the elementary particle physics and relativistic quantum mechanics are discussed. The relation with dark matter has implications for $\dot{J}$ vs. $\mathrm{M}^{2}$ plot of astronomical objects and the $w$-parameter.
\end{abstract}

Keywords: Medium, Vacuum polarization, Electrostatic potential energy, Angular momentum.

\section{Resumo}

Um exame do fenômeno físico da polarização do vácuo fornece suporte para o efeito das propriedades do meio na propagação de partículas carregadas e luz. As implicações para a física de partículas elementares e mecAnica quântica relativística são discutidas. A relação com a matéria escura tem implicações no gráfico $J$ vs. $M^{2}$ de objetos 'astronômicos e no parâmetro 'w.

Palavras-chave: Meio; Polarização do vácuo, Energia potencial eletrostática, Momento angular. 


\section{Introduction}

The equivalence principle is complemented by the postulated constancy of the speed of light in different frames of reference. However, vacuum polarization and $\breve{C}$ erenkov radiation, with the restriction on the angle of the coherent radiation emitted by a charged particle travelling faster than $\frac{c}{n}$, where $n$ is the refractive index and vacuum polarization $^{(1)}$, are suggestive of additional theory for propagation of systems in a medium.

While the factors in Lorentz transformations were derived by the invariance group of the Maxwell equations and the null cones, representing the directions of past and future wavefronts ${ }^{(2-5)}$, these factors in vacuum have been alternatively explained through contact of the photon with elements of a medium. The FitGerald-Lorentz factor $^{(2,13,14)}$ for the contraction of lengths in moving systems was derived initially through two different approaches to the propagation of light. Invariance of the Maxwell equations under a group of transformations of the electric displacement and magnetic fields and the time and spatial coordinates. It was demonstrated that the formulae for the displacement and magnetic fields had the same form in the new coordinates as the functional dependence of the original fields in the first set of coordinates. Evaluation of the momentum of the elecromagnetic system in the new coordinates and the acceleration, and equality between the electric and intertial masses, led to an expression to an equation for the multiplicative factor satisfies

$$
\frac{d(\gamma \cdot v)}{d v}=\gamma^{3}
$$

which has the solution $\gamma=\frac{1}{\left(1-v^{2}\right)^{\frac{1}{2}}}$. It followed that distances in the direction of the motion of the system were contracted through multiplication by a factor of $\frac{1}{\gamma}$, whereas perpendicular distances were unaffected.

Similarly, combining the theory of gyrostats of Kelvin with a postulate of the medium, through which an object moves, the the electromagnetic forces on the electrons in the medium would cause the body moving with uniform velocity $v$ to be contracted by a factor of $\frac{1}{\left(1-v^{2} / c^{2}\right)^{\frac{1}{2}}}$ in the direction of motion. Postulating the existence of an aether with a potential, the effect on a material system could be computed through the integral solutions to the electromagnetic equations.

The electric flux and the aether strain were combined in a modified form of Maxwell's equations, determining the motion of particles in the medium. One of the problems of the model was the constitution of the aether by charged particles, as the electrostatic energy would be too large for the Universe to have an $\Omega=1$ cosmology, and this paradox shall be outlined in $\S 3$ and resolved in $\S 5$. Nevertheless, the theory not only yielded the contraction factor but also a physical cause for this effect. While it may be suggested that it is not necessary to introduce any new physical hypotheses, the advantage of a tangible basis for the effect becomes clear at the quantum level.

This result, together with the interpretation of light as an electromagnetic wave, has led to the a theory of the motion of photons in a medium that has been characterized as aether consisting of charged particles ${ }^{(6-10)}$. It will be demonstrated that an identification of the aether with charged dark matter would yield an electrostatic potential energy that is $\mathcal{O}\left(10^{42}\right)$ times larger than that of the observed Universe. This dilemma may be resolved by identifying the aether with vacuum polarization and clouds of virtual particle-antiparticle pairs. For example, while electron-positron pairs are effectively neutral, the effect of the medium through the Lorentz factors still can be given a theoretical basis.

This model is consistent with relativistic theory with Lorentz transformations of four-vectors in space-time and the speed of light derived from the parameters of the electromagnetic field. Furthermore, the problem of the electrostatic potential energy is solved and a derivation of the gradients of the $J$ vs. $M^{2}$ plots follows from the identification of the identification of the angular momentum generated by the dark matter in addition to that of the gravitationally interacting matter.

The effect of a nontrivial quantum electrodynamic vacuum on the propagation of light has been evaluated ${ }^{(11)}$, yielding a general formula based on the scale anomaly of the Lagrangian ${ }^{(12)}$. The effects are known not to be currently measurable, although it has been established that velocities are decreased for a low magnetic field and strong magnetic fields ${ }^{(12)}$, and the effects are detectable. These results confirm the effect of the medium on the light propagation as well as that of charged particles.

\section{The Computation of the Slope of the $J$ vs. $M^{2}$ Plot for Astrophysical Objects}

The estimate of the electrostatic potential energy of dark matter consisting of charged particles will be based on a value for the energy that can be derived from the $J$ vs. $M^{2}$ plots for astronomical objects $(15,16,17)$. With a weighted average of $\alpha_{\text {het }}^{\prime}$, the heterotic string slope for gravitationally interacting systems and $\alpha_{\text {elec }}^{\prime}$, the value of $\alpha^{\prime}$ at astrophysical objects can be determined. Given that

$$
\begin{aligned}
\mu_{\text {het }} & =\frac{g^{2} c^{2}}{32 \pi^{2} G} \\
& =3.166286989 \times 10^{-3} g^{2} c^{2} / G
\end{aligned}
$$

it follows that

$$
\begin{aligned}
\alpha_{\text {het }}^{\prime} & =\frac{c}{2 \pi \mu_{\text {het }}} \\
& =\frac{c}{2 \pi\left(3.166286989 \times 10^{-3} g^{2} c^{2} / G\right)} \\
& =5.026548245 \times 10^{2} G / c
\end{aligned}
$$


The percentages attributed to the matter arising from the fields in the fundamental heterotic string effective action and dark matter in the Universe refer in the lowenergy limit to the gravitational potential energies measured in this proportion. Since the gravitational potential energy of two masses is $\frac{G M_{1} M_{2}}{r}$ and equals $\frac{G M^{2}}{r}$ for equal masses, the percentages correspond not to the masses but $M^{2}$. Suppose then that the purely gravitationally interacting matter satisfies

$$
J_{\text {grav }}=\alpha_{\text {grav }}^{\prime} M_{\text {grav }}^{2}
$$

and the angular momentum of the electon-positron pairs

$$
J_{\text {elec }}=\alpha_{\text {elec }}^{\prime} M_{\text {elec }}^{2}
$$

Then the angular momentum of an object consisting of both types of matter in proportion $c_{1}$ and $c_{2}$ is

$$
\begin{aligned}
J_{\text {ast }}= & \alpha_{\text {grav }}^{\prime}\left(M^{2}\right)_{\text {grav }}+\alpha_{\text {elec }}^{\prime}\left(M^{2}\right)_{\text {elec }} \\
= & \alpha_{\text {grav }}^{\prime}\left(c_{1}\left(M^{2}\right)_{\text {ast }}\right)+\alpha_{\text {elec }}^{\prime}\left(c_{2}\left(M^{2}\right)_{\text {ast }}\right) \\
& \left(c_{1} \alpha_{\text {grav }}^{\prime}=c_{2} \alpha_{\text {elec }}^{\prime}\right)\left(M^{2}\right)_{\text {ast }}
\end{aligned}
$$

which equals $\alpha_{a s t}^{\prime}\left(M^{2}\right)_{a s t}$ only if

$$
c_{1} \alpha_{\text {grav }}^{\prime}+c_{2} \alpha_{\text {elec }}^{\prime}=\alpha_{a s t}^{\prime} .
$$

The last equation is equivalent to

$$
\begin{aligned}
c_{1} \alpha_{\text {het }}^{\prime} & +c_{2} \alpha_{\text {elec }}^{\prime}=c_{1}\left(5.026548245 \times 10^{2} G / c\right) \\
& +c_{2}\left(1.3703599911 \times 10^{2} G / c\right) \\
c_{1} & +c_{2}=1
\end{aligned}
$$

which has the solution

$$
\begin{aligned}
& c_{1}=0.719229926 \\
& c_{2}=0.280740074
\end{aligned}
$$

The percentages of neutral gravitationally interacting matter and dark matter agree excellently with the observed values, which provides support for this model.

The potential paradox of a electron-positron pair, being neutral in its entirety, and the use of the electromagnetic parameter $\alpha_{\text {elec }}^{\prime}$ shall be resolved in $\S 5$. The entire angular momentum is constructed by the addition of infinitesimal angular momentum vectors in the medium.

\section{A Calculation of the Electrostatic Potential En- ergy of Charged Dark Matter}

The measured energy densities of the baryons, dark matter and dark energy have been estimated to be and $70 \pm 10 \%{ }^{(18-20)}$ Amongst the viable cosmological models currently is the $\Lambda C D M$ model, with the dark energy identified with $\Lambda$ term $^{(19)}$.
Suppose that the fraction of the squared mass that is derived from the original Lagrangian field theory and interacts gravitationally is $71.9229926 \%$, whereas $\Omega_{C D M}=0.280740074$. The energy of the Universe is $1.148814903 \times 10^{90} \mathrm{eV}$ from the critical density and the comoving radius ${ }^{(21)}$. Consequently, the corresponding gravitational potential energy of the dark matter would be

$$
\begin{aligned}
& \left(\frac{0.280740074}{0.719229926}\right)\left(1.418814903 \times 10^{90} \mathrm{eV}\right) \\
= & 5.538120516 \times 10^{89} \mathrm{eV}
\end{aligned}
$$

The spherical volume of Universe with a comoving radius of $4.3353942 \times 10^{26} \mathrm{~m}$ is

$$
\begin{array}{ll}
\frac{4 \pi}{3} & \left(4.3353942 \times 10^{26} \mathrm{~m}\right)^{3} \\
= & 3.413299413 \times 10^{80} \mathrm{~m}^{3} \\
= & 3.413299413 \times 10^{110} \AA^{3}
\end{array}
$$

Suppose initially that the dark matter consists of electrons and that the number equals $N_{e^{-} \text {dark matter. Given }}$ that only half of the Universe can be observed, the average volume occupied by an electron is

$$
\begin{aligned}
& \frac{1}{2} \frac{3.413299413 \times 10^{110} \AA^{3}}{N_{e^{-}, \text {dark matter }}} \\
= & \frac{1.706649707 \times 10^{110} \AA^{3}}{N_{e^{-}, \text {dark matter }}}
\end{aligned}
$$

while the mean distance between the electrons is then

$$
\begin{aligned}
& \left(\frac{3}{4 \pi} \frac{1.706649707 \times 10^{110} \AA^{3}}{N_{e^{-}, \text {dark matter }}}\right)^{\frac{1}{3}} \\
= & \frac{3.44104657 \times 10^{36} \AA}{N_{e^{-}, \text {dark matter }}}
\end{aligned}
$$

If the electrons are arranged in a three-dimensional array, with the number of sites at each level being $18 \ell^{2}-6 \ell$,

$$
\begin{aligned}
\sum_{\ell=1}^{\ell_{\max }}\left(18 \ell^{2}-6 \ell\right) & =3 \ell_{\max }\left(\ell_{\max }+1\right)\left(2 \ell_{\max }+1\right) \\
& -3 \ell_{\max }\left(\ell_{\max }+1\right)
\end{aligned}
$$

and the number of levels is

$$
\ell_{\max }=\left(\frac{1}{6} N_{e^{-}, \text {dark matter }}\right)^{\frac{1}{3}}
$$


The sum of the reciprocal distances then becomes

$$
\begin{aligned}
\sum_{\ell=1}^{\ell_{\max }} & \left(\frac{4.335265245 \times 10^{36} \AA}{N_{e^{-}}, \text {dark matter }} \ell\right)^{-1} \times \\
& \left(18 \ell^{2}-6 \ell\right) \\
= & \left(\frac{4.335265245 \times 10^{36} \AA}{N_{e^{-}}, \text {dark matter }}\right)^{-1} \times \\
& \left(9 \ell_{\max }^{2}+3 \ell_{\max }\right) \\
= & \left(4.335265245 \times 10^{36} \AA\right)^{-1} \times \\
& {\left[\frac{9}{6^{\frac{2}{3}}} N_{e^{-}}^{\frac{5}{3}}, \text { dark matter }+\frac{3}{6^{\frac{1}{3}}} N_{e^{-}}^{4^{\frac{4}{3}}, \text { dark matter }}\right] }
\end{aligned}
$$

The gravitational potential energy of charged dark matter would be

$$
\begin{aligned}
& \left(6.682669 \times 10^{-57}(\mathrm{eV})^{-2}\right) \cdot \\
& \frac{N_{e^{-}}, \text {dark matter }}{2} \cdot(0.511 \mathrm{MeV})^{2} . \\
& \left(4.335265245 \times 10^{36} \AA\right)^{-1} \cdot \\
& \left(1.9752479 \times 10^{3} \mathrm{eV}-\AA\right) \cdot \\
& {\left[\frac{9}{6^{\frac{2}{3}}} N_{e^{-}}^{\frac{5}{3}}, \text { dark matter }+\frac{3}{6^{\frac{1}{3}}} N_{e^{-}, \text {dark matter }}^{\frac{4}{3}}\right] } \\
\approx & \left(2.16707 \times 10^{-78} \mathrm{eV}\right) N_{e^{-}}^{\frac{8}{3}} \text {, dark matter } \\
= & 3.983182 \times 10^{89} \mathrm{eV}
\end{aligned}
$$

It follows that

$$
\begin{aligned}
N_{e^{-}, \text {dark matter }}^{\frac{8}{3}} & =\frac{3.983182 \times 10^{89} \mathrm{eV}}{2.16707 \times 10^{-78} \mathrm{eV}} \\
& \doteq 1.8380495 \times 10^{167}
\end{aligned}
$$

and

$$
N_{e^{-}, \text {dark matter }} \approx 5.29 \times 10^{62}
$$

To determine the electrostatic potential energy of the dark matter, with

$$
\begin{aligned}
k & =8.897 \times 10^{9} \frac{N m^{2}}{C^{2}} \\
e & =1.6022 \times 10^{-19} C \\
k e^{2} & =14.39917514 \mathrm{eV}-\AA
\end{aligned}
$$

it can be approximated, for a uniform distribution, by

$$
\begin{aligned}
& (14.399175514 \mathrm{eV}-\stackrel{\AA}{A}) \cdot \\
& \frac{\left(5.29 \times 10^{62}\right)^{2}}{2} \\
& \left(4.335265245 \times 10^{36} \AA\right)^{-1} . \\
& \frac{9}{6^{\frac{2}{3}}}\left(5.29 \times 10^{62}\right)^{\frac{5}{3}}
\end{aligned}
$$

The electrostatic potential energy of uniformly distributed charged then would be $8.32 \times 10^{141} \mathrm{eV}$. Other distributions of the charge also yield values which are too large. The effect of the configuration of the charges on the potential energy is examined in Appendix B. For sufficiently low velocities, the kinetic energy of the constituents of the dark matter would not add significantly to the energy of the electromagnetic field.

Since the electrostatic potential energy of dark matter consisting of a uniform distribution of electrons is $\mathcal{O}\left(10^{141} \mathrm{eV}\right)$, whereas the observed energy of the dark matter in the universe is $\mathcal{O}\left(10^{89} \mathrm{eV}\right)$, the upper limit on the fraction of charged particles in the dark matter must be

$$
\mathcal{O}\left(\left(\frac{3.983182 \times 10^{89} \mathrm{eV}}{8.32 \times 10^{141} \mathrm{eV}}\right)^{\frac{3}{8}}\right)=\mathcal{O}\left(10^{-20}\right) .
$$

Therefore, the model of aether consisting of charged particles must be changed to one with primarily neutral objects to obtain consistency with the energy of the observed Universe. It has been suggested further that various types of matter such as neutrinos, the supersymmetric partners or axions could could contribute to the dark matter. While these models are consistent with the zero net charge of almost all of the constitutents of dark matter, the computation in $\S 2$ shows that an essentially electrical component is necessary. The prediction of the slope of the $J$ vs. $M^{2}$ plots would not be a property of the approaches based on neutrinos, supersymmetric partners and axions because of the neutrality of these particles, which also not postulated to have a substructure.

The resolution of the theoretical paradox implied by the two physical conditions following from the discussion in $\S 2$ and this section is provided by virtual electronpositron pairs resulting from vacuum polarization. This model of dark matter is supported by a conjectured solution to the potential problem of density cusps at the cores of galaxies through the annihilation ${ }^{(22)}$

Investigations of the dark energy have been related to the $w$ parameter, where $w=\frac{\langle p\rangle}{\langle\rho\rangle}$, and other statefinder parameters ${ }^{(23)}$. Observations of the cosmological acceleration of galaxies reveals that $-1.4<w<-0.65$ for $0.15<\Omega_{m}<0.4^{(24-26)}$. The use of vacuum energy as a model for dark energy also has been questioned ${ }^{(27)}$, based on the decrease of the magnitude of $\Lambda$ and the constancy of quantum fluctuations of the fields and the geometrical basis for a non-zero value of $\Lambda$ in certain geometries arising as solutions of gravitational field equations. Moreover, from the foregoing analysis, it would be not be feasible to identify both vacuum energy as the source of more than one major component of the total energy. Instead, given the existence of a large value of $\Lambda$ during the inflationary era, it is more likely that the dark energy may be traced to a scalar potential in a fundamental quantum theory such as heterotic string theory $(21,28)$. Even though it is preferable then to distinguish between the sources of dark matter and dark energy, quintessance 
models ${ }^{(29-31)}$ and theories of unification of these two types of energy ${ }^{(20)}$ utilize a generalized scalar field Lagrangian, which is consistent with the interpretation of the dark energy given here.

\section{The Creation and Annihilation of Particle- Antiparticle Pairs}

One of the postulates of special relativity, that all physical laws hold equally in different frames of reference, may be verified by the decay of elementary particles. Energy conservation prevents the decay of particles into products with rest masses that sum to a greater mass. However,this inequality is verified again in Appendix A if the initial particle has sufficient momentum.

The physical effect of particle-antiparticle pairs is confirmed by a semiclassical model of the electron-positron creation and annihilation. This two body system may be described by the equation

$$
2 \gamma m_{e} c^{2}+U(r)=0
$$

where $r$ is the distance from the axis of symmetry of one of the particles, $R=2 r$ is the distance between the particle and antiparticle and $U(r)$ is the sum of electric and magnetic components of the potential ${ }^{(32)}$

$$
U(r)=-\frac{e^{2}}{2 r}-2 \frac{\mu_{e}}{(2 r)^{3}} .
$$

At a maximum separation between the particle and antiparticle, $R_{\max }=2 r_{\max }, \gamma=1$ and

$$
2 m_{e} c^{2}-\frac{e^{2}}{R_{\max }}-2 \frac{\mu_{e}^{2}}{R_{\max }^{3}}=0 .
$$

An estimate of $R_{\max }$ can be derived by presuming that $R_{\text {max }}=\rho_{*} \cdot c t_{H e}$, with the interval for the virtual pair to exist by quantum mechanics being

$$
t_{H}=\frac{\frac{\hbar}{2}}{2 m c^{2}} .
$$

Since

$$
\mu_{e}=\frac{g e}{2 m_{e} c} \frac{\hbar}{2}=\frac{g}{2} \cdot 2 e c t_{H e}
$$

where $g$ is the Landé factor and

$$
\frac{U_{B}}{V_{e}}=2 \frac{\mu_{e}^{2}}{e^{2}} \frac{1}{R^{2}}=8 \cdot\left(\frac{g}{2}\right)^{2}\left(\frac{c t_{H e}}{R}\right)^{2}
$$

it follows that, at maximum seperation,

$$
2 m_{e} c^{2}+\left[1+\frac{\rho_{*}^{2}}{8\left(\frac{g}{2}\right)^{2}}\right] \cdot\left(-2 \frac{\mu_{e}^{2}}{R_{\max }^{3}}\right)=0
$$

and

$$
\begin{aligned}
R_{\max } & =\left(\frac{\mu_{e}^{2}}{m_{e} c^{2}}\right)^{\frac{1}{3}}\left[1+\frac{\rho^{2}}{8\left(\frac{g}{2}\right)^{2}}\right]^{\frac{1}{3}} \\
& \approx(1.0625)^{\frac{1}{3}}\left(4.7 \times 10^{-14} \mathrm{~m}\right)
\end{aligned}
$$

if $\rho_{*} \approx \frac{1}{2}$. From Eq.(4.1),

$$
2 \gamma m_{e} c^{2}-\frac{e^{2}}{\gamma R}-2 \frac{\mu_{e}^{2}}{(\gamma R)^{3}}=0
$$

such that

$$
\gamma=\frac{\frac{e^{2}}{\gamma R}+\frac{2 \mu_{e}^{2}}{\gamma^{3} R^{3}}}{\frac{e^{2}}{R_{\max }}+\frac{2 \mu_{e}^{2}}{R_{\max }^{3}}}
$$

Given that

$$
\begin{gathered}
\frac{e^{2}}{R_{\max }}=\frac{\rho_{*}^{2}}{8\left(\frac{g}{2}\right)^{2}} \cdot \frac{2 \mu_{e}^{2}}{R_{\max }^{3}} \\
\frac{e^{2}}{\gamma R}=\frac{\rho(\gamma)^{2}}{8\left(\frac{g}{2}\right)^{2}} \cdot \frac{\mu_{e}^{2}}{\gamma R^{2}}, \\
\gamma^{4}=\frac{\left[1+\frac{\rho(\gamma)^{2}}{8\left(\frac{g}{2}\right)^{2}}\right]}{\left[1+\frac{\rho_{*}^{2}}{8\left(\frac{g}{2}\right)^{2}}\right]}\left(\frac{R_{\max }}{R}\right)^{3} .
\end{gathered}
$$

As

$$
\frac{\frac{e^{2}}{\gamma R}}{\frac{2 \mu_{e}^{2}}{(\gamma R)^{3}}}=\frac{\gamma^{2}}{8\left(\frac{g}{2}\right)^{2}} \cdot\left(\frac{R}{c t_{H e}}\right)^{2}
$$

If $\gamma^{2} \sim\left(\frac{R_{\max }}{R}\right)^{\frac{3}{2}}$,

$$
\begin{aligned}
\gamma^{2} \cdot R^{2} & \sim\left(\frac{R_{\max }^{\frac{3}{2}}}{R^{\frac{3}{2}}}\right) \cdot R^{2} \\
& =R^{\frac{1}{2}} R_{\max }^{\frac{3}{2}} \\
& \sim \gamma^{-\frac{2}{3}} R_{\max }^{2} .
\end{aligned}
$$

Consequently, the function $\rho(\gamma)$ can be estimated to be $\gamma^{-\frac{2}{3}}$ over over much of the trajectories of the particle and antiparticle. The maximum value of the multiplicative factor in Eq.(4.12) is 1, whereas the minimum values is $\left[1+\frac{\rho_{*}^{2}}{8\left(\frac{g}{2}\right)^{2}}\right]^{-1}$. As $\rho_{*} \approx \frac{1}{2}$,

$$
\left[1+\frac{\rho_{*}^{2}}{8\left(\frac{g}{2}\right)^{2}}\right]^{-1} \approx \frac{1}{1.0625}
$$

Let $\gamma^{2} \approx\left(\frac{1}{1.0625}\right)^{\frac{1}{2}}\left(\frac{R_{\max }}{R}\right)^{\frac{3}{2}}=\zeta^{-\frac{3}{2}}$ for the initial portion of the trajectory and $\rho(\gamma) \rightarrow \rho_{*}$ as $R \rightarrow R_{\max }$, with $\zeta^{-\frac{3}{2}} \simeq\left(\frac{R_{\max }}{R}\right)^{\frac{3}{2}}$ near the point of maximum of separation. Integrating

$$
d t= \pm \frac{d r}{c \sqrt{1-\zeta^{\frac{3}{2}}}}
$$

yields a semiclassical estimate of the time of the creation 
and annihilation process of

$$
\begin{aligned}
& t_{v e}^{(1)}=2 \cdot \frac{1}{c} \int_{0}^{\frac{1}{(1.0625)^{\frac{1}{3}}} \frac{R_{\max }}{2}} \\
& \frac{d r}{\sqrt{1-(1.0625)^{\frac{1}{2}}\left(\frac{R}{R_{\max }}\right)^{\frac{3}{2}}}} \\
& +2 \cdot \frac{1}{c} \int_{\frac{1}{(1.0625)^{\frac{1}{3}}} \frac{R_{\max }}{2}}^{\frac{R_{\max }}{(r}} \\
& \sqrt{1-\left(\frac{R}{R_{\max }}\right)^{\frac{3}{2}}}
\end{aligned}
$$

With the variable $\tilde{\zeta}=(1.0625)^{\frac{1}{3}} \zeta$,

$$
\begin{aligned}
t_{v e}^{(1)=} & \frac{R_{\max }}{(1.0625)^{\frac{1}{3}} c} \int_{0}^{1} \frac{d \tilde{\zeta}}{\sqrt{1-\tilde{\zeta}^{\frac{3}{2}}}}+ \\
& \frac{R_{\max }}{c} \int_{\frac{1}{(1.0625)^{\frac{1}{3}}}}^{1} \frac{d \zeta}{\sqrt{1-\zeta^{\frac{3}{2}}}}
\end{aligned}
$$

With the change of variables $w=1-\zeta^{\frac{3}{2}}$,

$$
\int_{0}^{1} \frac{d \zeta}{\sqrt{1-\zeta^{\frac{3}{2}}}}=\frac{2}{3} \int_{0}^{1} \frac{d w}{w^{\frac{1}{2}}(1-w)^{\frac{1}{3}}}
$$

and, setting $w=\sin ^{2} \theta$,

$$
\frac{2}{3} \int_{0}^{1} \frac{d w}{w^{\frac{1}{2}}(1-w)^{\frac{1}{3}}}=\frac{4}{3} \int_{0}^{\frac{\pi}{2}}(\cos \theta)^{\frac{1}{3}} d \theta
$$

yielding an equality with trigonometric integral. Furthermore,

$$
\int_{\frac{1}{(1.0625)^{\frac{1}{3}}}}^{1} \frac{d \zeta}{\sqrt{1-\zeta^{\frac{3}{2}}}} \approx \frac{4}{3} \int_{0}^{\arcsin \left[(0.0298575)^{\frac{1}{2}}\right]}(\cos \theta)^{\frac{1}{3}} d \theta
$$

Let

$$
T(b)=\int_{0}^{b}(\cos \theta)^{\frac{1}{3}} d \theta
$$

such that the Taylor series is

$$
\begin{aligned}
T(b) & =T(0)+T^{\prime}(0) b+\frac{T^{\prime \prime}(0)}{2 !} b^{2}+\frac{T^{\prime \prime \prime}(0)}{3 !} b^{3}+\ldots \\
& =b-\frac{1}{18} b^{3}+\ldots
\end{aligned}
$$

and

$$
T(0.173665) \approx 0.173374
$$

Then

$$
\begin{aligned}
t_{v e}^{(1)} & \approx 2.71788 \times 10^{-22} \sec (1.0625)^{\frac{1}{3}} \\
& {\left[\frac{1}{(1.0625)^{\frac{1}{3}}}+\frac{4}{3} \frac{0.173374}{1.7247}\right] } \\
\doteq \quad & \left(2.71788 \times 10^{-22} \mathrm{sec}\right)(1.0625)^{\frac{1}{3}}(1.1140268) \\
= & 3.0894897 \times 10^{-22} \mathrm{sec}
\end{aligned}
$$

In string theory, the paths are represented by surfaces defining the super-worldsheet trajectories. Replacing the point-particles by strings and using the effective closed string coupling of $\frac{1}{24.35097904}$, The average time for a real process would be given by a weighted sum over the genus

$\alpha_{1} t_{v e}^{(1)}+\frac{1}{24.35097904}\left(2 t_{v e}^{(1)}\right)+\frac{1}{(24.35097904)^{2}}\left(3 t_{v e}^{(1)}\right)+\ldots$

because the splitting and rejoining of strings in a genustwo surface should occur in twice the time as that of a one-loop process, $t_{v e}^{(1)}$. The coefficient $\alpha_{1}$ can be chosen such that the sum of the coefficients equals 1 . While a weighted average over the space of super-worldsheets could be included, this supermoduli space also may be identified with the space of metrics modulo diffeomorphisms and Weyl rescalings, implying that the time variable can be adjusted to the original value through a change of affine parameter, and there would be a cancellation of supermoduli space integrals.

$$
\alpha_{1}=\left[\frac{1-\frac{2}{24.35097904}}{1-\frac{1}{24.35097904}}\right]
$$

The average time equals

$$
\begin{aligned}
& t_{v e}=\left(\alpha_{1}+0.0874835\right) t_{v e}^{(1)} \doteq \\
& (0.95717303+0.08748808) t_{v e} \\
& =1.04466111 t_{v e}^{(1)}
\end{aligned}
$$

From Eq.(4.25), it follows that

$$
\begin{aligned}
& t_{v e} \approx(1.04466111)(3.0894897 \times \\
& \left.10^{-22} \text { sec }\right) \doteq 3.2274697 \times 10^{-22} \mathrm{sec}
\end{aligned}
$$

which compares well with the bound derived from the uncertainty relation.

\section{The Quantum Theory of Light and Vacuum Po- larization}

Since quantum field theory represents a unification of relativity and quantum mechanics within a functional analytic framework, its predictions are supported by the validity of the Lorentz invariance at the quantum level. Amongst the predictions of quantum field theory is the occurrence of virtual particle-antiparticle pairs in the vacuum. The initial effect of the particle-antiparticle pairs is the change in the strength of the force through renormalization of the coupling. In addition to dependence of the coupling on the energy, it can be established that the potential is effectively modified as a result of this renormalization. This is particularly relevant to the quantum theory of gravity where the problem of the curvature singularity is expected to be resolved. 
It remains to be shown that the conclusions of the theory of aether on the speed of light, the FitzGeraldLorentz factors, while providing a resolution of the problem of the electromagnetic potential energy of the aether, are also consistent with the conclusions of $\S 2$ regarding the slope of the $J$ vs. $M^{2}$ plot for astronomical objects. The velocity of light is calculated again from the ratio of the coefficients of potential and kinetic energy integrals resulting from the generalized Maxwell equations. It has been shown that the potential energy of the aether is

$$
E_{p o t}=\frac{1}{2} B \int\left(f^{2}+g^{2}+h^{2}\right) d \tau
$$

where $(f, g, h)$ is the electric displacement of the $\operatorname{aether}^{(9)}$.

Although an electron-positron pair has zero total charge, it possesses a moment, and therefore angular momentum, as a result of the pair being an electromagnetic couple. Furthermore, the moment would be $F d$, where $F$ is the magnitude of the force exerted by the electron or positron and $d$ is the distance between the electron and positron, which then would equal the $\frac{1}{e \epsilon_{0}}(f, g, h)$. A couple and a coplanar force are equaivalent to the action of a force at a distance thereby introducing angular momentum to the system. Consequently, the virtual pair is precisely the object to create a rotational energy that is equal to the potential energy of the aether (5.1) because it is proportional to $I^{2}$, where $I$ is the moment of inertia.

As the photon propagates in the original theory through contact with the electron, this theory may be rephrased when the medium contains electron-positron pairs. The photon then must have local interactions with the electrons and positrons in vertex diagrams, and the propagation of the states through further contact gives rise to addition of the angular momentum vectors and a contribution to the rotation of astronomical objects. Consequently, this generalization of the original model is consistent with the current experimental evidence.

\section{Appendices}

\section{The Creation of Final State Pairs from an Initial State with Momentum}

Energy and momentum conservation

$$
\begin{aligned}
E & =E_{1}+E_{2} \\
\vec{p} & =\vec{p}_{1}+\vec{p}_{2}
\end{aligned}
$$

The momentum conservation relation implies that

$$
\vec{p} \cdot \vec{p}=\left|\vec{p}_{1}\right|^{2}+\left|\vec{p}_{2}\right|^{2}+2 \vec{p}_{1} \cdot \vec{p}_{2}
$$

and

$$
E=\sqrt{|\vec{p}|^{2}+m^{2}}=\sqrt{\left|\vec{p}_{1}\right|^{2}+m_{1}^{2}}+\sqrt{\left|\vec{p}_{2}\right|^{2}+m_{2}^{2}}
$$

It is now intended to determine the maximum value of $m_{1}+m_{2}$ Squaring Eq.(A.3) gives

$$
\begin{aligned}
|\vec{p}|^{2}+m^{2} & =\left|\vec{p}_{1}\right|^{2}+m_{1}^{2}+\left|\vec{p}_{2}\right|^{2}+m_{2}^{2} \\
& +2 \sqrt{\left|\vec{p}_{1}\right|^{2}+m_{1}^{2}} \sqrt{\left|\vec{p}_{2}\right|^{2}+m_{2}} \\
2 \vec{p}_{1} \cdot \vec{p}_{2} & =m_{1}^{2}+m_{2}^{2} \\
& +2 \sqrt{|\vec{p}|^{2}+m_{1}^{2}} \sqrt{\left|\vec{p}_{2}\right|^{2}+m_{2}^{2}}
\end{aligned}
$$

Suppose that $\vec{p}_{1}$ and $\vec{p}_{2}$ are chosen to be collinear such that $\vec{p}_{1} \cdot \vec{p}_{2}=\left|\vec{p}_{1}\right|\left|\vec{p}_{2}\right|$. For this reaction,

$$
\begin{aligned}
& \left(m_{1}+m_{2}\right)^{2}=m^{2}+2\left|\vec{p}_{1}\right|\left|\vec{p}_{2}\right|+ \\
& 2\left[m_{1} m_{2}-\sqrt{\left|\vec{p}_{1}\right|^{2}+m_{1}^{2}} \sqrt{\left|\vec{p}_{2}\right|^{2}+m_{2}^{2}}\right]
\end{aligned}
$$

Consider now the inequality

$$
\left|\vec{p}_{1}\right|\left|\vec{p}_{2}\right|-\sqrt{\left|\vec{p}_{1}\right|^{2}+m_{1}^{2}} \sqrt{\left|\vec{p}_{2}\right|^{2}+m_{2}^{2}}+m_{1} m_{2} \leq 0
$$

This would imply that

$$
\begin{aligned}
& \left|\vec{p}_{1}\right|^{2}\left|\vec{p}_{2}\right|^{2}+m_{1}^{2} m_{2}^{2}+2\left|\vec{p}_{1}\right|\left|\vec{p}_{2}\right| m_{1} m_{2} \leq \\
& \left|\vec{p}_{1}\right|^{2}\left|\vec{p}_{1}\right|^{2}+m_{1}^{2} m_{2}^{2}+\left|\vec{p}_{1}\right|^{2} m_{2}^{2}+\left|\vec{p}_{2}\right|^{2} m_{1}^{2}
\end{aligned}
$$

or

$$
\left|\vec{p}_{1}\right|^{2} m_{2}^{2}+\left|\vec{p}_{2}\right|^{2} m_{1}^{2}-2\left|\vec{p}_{1}\right|\left|\vec{p}_{2}\right| m_{1} m_{2} \geq 0
$$

which is clearly valid. 


\section{The Effect of the Distribution of Charges on the Electrostatic Potential Energy}

Consider a uniform distribution of charges. The change in the potential energy with respect to a change in the position of a single charge is

$$
\begin{aligned}
\delta V & =\frac{\partial V}{\partial x} \delta x+\frac{\partial V}{\partial y} \delta y+\frac{\partial V}{\partial z} \delta z \\
& =-F_{x} \delta x-F_{y} \delta y-F_{z} \delta z
\end{aligned}
$$

Since $V=\sum_{i<j} \frac{e^{2}}{r_{i j}}, F_{i j}=\frac{e^{2}}{r_{i j}^{2}}$, and, in the coordinate directions, the forces are

$$
\begin{aligned}
& F_{i j x}=\frac{e^{2}}{r_{i j}^{3}} x_{i j} \\
& F_{i j y}=\frac{e^{2}}{r_{i j}^{3}} y_{i j} \\
& F_{i j z}=\frac{e^{2}}{r_{i j}^{3}} z_{i j}
\end{aligned}
$$

Suppose that the positions of the charges are projected to the coordinate axes. Then, it follows that there is an ordering of the $x$-coordinates, for example, and the charges may be labelled according to this $x$-coordinate. The following constraint in an approximately cubical configuration

$$
\sum_{\ell_{x}=1}^{N-1} x_{i_{\ell_{x}}, i_{\ell_{x}}+1}=V^{\frac{1}{3}}
$$

and similarly, with the labelling defined by $y$ and $z$ coordinates,

$$
\begin{aligned}
& \sum_{\ell_{y}=1}^{N-1} y_{i_{\ell_{y}}, i_{\ell_{y}}+1}=V^{\frac{1}{3}} \\
& \sum_{\ell_{z}=1}^{N} z_{i_{\ell_{z}}, i_{\ell_{z}}+1}=V^{\frac{1}{3}}
\end{aligned}
$$

The forces and the change in the electrostatic potential energy can be evaluated subject to these conditions for each coordinate direction. It is useful, therefore, to consider a linear set of charges. For three charges, with the distance from the edge to the center being $r$, the potential energy is $\frac{5 e^{2}}{2 r}$. If the central charge is moved to the right by $\delta r$, the potential energy becomes

$$
\begin{aligned}
\frac{e^{2}}{r-\delta r}+\frac{e^{2}}{r+\delta r}+\frac{e^{2}}{2 r} & =\frac{2 e^{2} r}{r^{2}-(\delta r)^{2}}+\frac{e^{2}}{2 r} \\
& >\frac{5 e^{2}}{2 r}
\end{aligned}
$$

The potential energy of $n$ charges evenly distributed along a line is

$$
\begin{aligned}
& \frac{e^{2}}{r}+\frac{e^{2}}{2 r}+\ldots+\frac{e^{2}}{(n-1) r} \\
+ & \frac{e^{2}}{r}+\frac{e^{2}}{2 r}+\ldots+\frac{e^{2}}{(n-2) r} \\
+ & \ldots \\
+ & \frac{e^{2}}{r} \\
= & {\left[(n-1)+\frac{n-2}{2}+\frac{n-3}{3}\right]+\ldots } \\
+ & {\left[\frac{2}{n-2}+\frac{1}{n-1}\right] \frac{e^{2}}{r} }
\end{aligned}
$$

Defining the function $v(n)=n+\frac{n-1}{2}+\ldots+\frac{1}{n}, v(2)=\frac{5}{2}$, $v(3)=\frac{13}{3}, v(4)=\frac{77}{12}$ and

$$
\begin{aligned}
& v(n-1)=n-1+\frac{n}{2}-1+\frac{n}{3}-1+\ldots \\
& +\frac{n}{n-1}-1 \\
& =n\left(1+\frac{1}{2}+\ldots+\frac{1}{n-1}\right)-(n-1) \\
& =n \psi(n-1)-(n-1)
\end{aligned}
$$

$v(n) \approx(n+1) \ln n-n$

If an interior charge located a distance $\ell r$ from the leftmost charge is shifted by $\delta r$, the electrostatic potential energy becomes

$$
\begin{aligned}
& \frac{e^{2}}{r}+\ldots+\frac{e^{2}}{\ell r+\delta r}+\ldots+\frac{e^{2}}{(n-1) r} \\
+ & \frac{e^{2}}{r}+\ldots+\frac{e^{2}}{(\ell-1) r+\delta r}+\ldots+\frac{e^{2}}{(n-2) r} \\
& \vdots \\
+ & \frac{e^{2}}{r}+\frac{e^{2}}{2 r}+\ldots+\frac{e^{2}}{(n-\ell-2) r} \\
+ & \frac{e^{2}}{r}
\end{aligned}
$$

Comparing $\frac{e^{2}}{\ell r}+\frac{e^{2}}{(\ell-1) r}+\ldots+\frac{e^{2}}{r}+\frac{e^{2}}{r}+\frac{e^{2}}{2 r}+\ldots+\frac{e^{2}}{(n-1-\ell) r}$ with

$\frac{e^{2}}{\ell r+\delta r}+\frac{e^{2}}{(\ell-1) r+\delta r}+\ldots+\frac{e^{2}}{r+\delta r}+\frac{e^{2}}{r-\delta r}+\frac{e^{2}}{2 r-\delta r}+\ldots+$ $\frac{e^{2}}{(n-1-\ell) r-\delta r}$, the second expression is larger when $n-$ $1 \geq 2 \ell$ and $\delta r>0$. If $n-1<2 \ell$, the distances may be measured from the rightmost charge, and the same conclusion is reached if the charge is moved to the left by a positive amount. However, if $n-1<2 \ell$ and the charge is moved to the right by $\delta r>0$, the electrostatic potential energy is lowered. For example, a shift of the third charge in a configuration of four charges yields the potential energy

$$
\frac{11}{6} \frac{e^{2}}{r}+\frac{e^{2}}{2 r+\delta r}+\frac{2 e^{2} r}{r^{2}-(\delta r)^{2}}
$$


An expansion in $\frac{\delta r}{r}$ of

$$
\frac{e^{2}}{2 r+\delta r}+\frac{2 e^{2} r}{r^{2}-(\delta r)^{2}}-\frac{e^{2}}{2 r}-\frac{2 e^{2}}{r}
$$

gives

$$
\begin{aligned}
& -\frac{e^{2}}{4 r}\left(\frac{\delta r}{r}\right)+\frac{17 e^{2}}{8 r}\left(\frac{\delta r}{r}\right)^{2}-\frac{e^{2}}{16 r}\left(\frac{\delta r}{r}\right)^{3}+ \\
& \frac{65}{32} \frac{e^{2}}{r}\left(\frac{\delta r}{r}\right)^{4}-. .
\end{aligned}
$$

For $\frac{\delta r}{r} \ll \frac{1}{8}$, this sum is negative, and the electrostatic energy is lowered, which is also consistent with the estimate of the forces on the charge. Amongst the configurations of four charges, with only the third charge being shifted, the minimum value of the electrostatic potential energy is approximately

$$
\left(\frac{13}{3}-\frac{1}{136}\right) \frac{e^{2}}{r}
$$

This analysis can be extended to the $y$ and $z$ coordinates and three-dimensional arrays, It follows that the potential energy of the uniformly distribution of charges is not significantly different from the global minimum represented by a slightly perturbed configuration.

\section{References}

1. SEN GUPTA, N. D.: On the energy-loss in the Cerenkov Radiation, Annalen Phys. 28, 211-215 (1972).

2. LORENTZ, H. A.: De relatieve beweging van de aarde en den aether, Koninklijke Akademie van Wetenschappen te Amsterdam, Wissen Natuurkundige Afdeeling. Verslagen der Zittingen 1, 74-79 (1892-1893).

3. LORENTZ, H. A.: Versuch einer theorie der electrischen und optischen erscheinungen in bewegten körpere, Brill, Leyden (1895).

4. LORENTZ, H. A.: Simplified theory of electrical and optical phenomena in moving systems, Proc. Acad. Science Amsterdam I, 427-433 (1899).

5. LORENTZ, H. A.: Electromagnetic phenomena in a system moving with any Velocity less than that of light, Proc. Acad. Science Amsterdam IV, 669-678 (1904).

6. LARMOR, J.: A dynamical theory of the electric and luminiferous medium, Proc. Roy. Soc. Lond. 54, 438-461 (1893).

7. LARMOR, J.: A dynamical theory of the electric and luminiferous medium, Part II; a theory of electrons, Proc. Roy. Soc. Lond. 58, 222-228 (1895).

8. LARMOR, J.: A dynamical theory of the electric and luminiferous medium, Part III; relations with material media,
Proc. Roy. Soc. Lond. 61 272-285 (1897).

9. LARMOR, J.: Aether and Matter, Cambridge University Press, Cambridge (1900).

10. LARMOR, J.: On the ascertained absence of effects of motion through the aether in relation to the constitution of matter and on the FitzGerald-Lorentz hypothesis, Proc. Phys. Soc. London 19, 253-258 (1903).

11. LATORRE, J. I., PASCUAL, P., TARRASCH, R.: Speed of light in non-trivial vacua, Nucl. Phys. B437, 60-82 (1995).

12. DITTRICH, W., GIES, H.: Light propagation in nontrivial QED vacua, Phys. Rev. D58, 025004:1-13 (1998).

13. FITZGERALD, G. G.: The ether and the Earth's atmosphere, Science 13, 390 (1889).

14. HEAVISIDE, O.: The electro-magnetic effects of a moving charge, Electrician 22, 147-148 (1888).

15. WESSON, P. S.: Clue to the unification of gravitation and particle physics, Phys. Rev. D23, 1730-1734 (1981).

16. TASSIE, L.: The formation of the universe from rotating superstrings, Aust. J. Phys. 40, 109-116 (1987).

17. BURDEN, C. J. and Tassie, L.: Rotating strings, glueballs and exotic mesons, Aust. J. Phys. 35, 223-233 (1982).

18. TURNER, M. S.: Dark energy and energy in the universe, Phys. Scripta T85, 210-220 (2000).

19. SAHNI, V.: Dark matter and dark energy, Lect. Notes Phys. 653, 141-180 (2004).

20. SCHERRER, R. J.: Purely kinetic $k$-Essence as unified dark matter, Phys. Rev. Lett. 93, 011301:1-5 (2004).

21. DAVIS, S.: Energy in the very early universe, Phys. Lett. B64, 299-309 (2007).

22. KAPLINGHAT, M., KNOX, L., TURNER, M. S.: Annihilating dark matter, Phys. Rev. Lett. 85, 3335-3338 (2000).

23. ZIMDAHL, W., PAVON, D.: Statefinder parameters for interacting dark energy, Gen. Rel. Grav. 36, 1483-1491 (2004).

24. HUTERER, D., TURNER, M. S.: Constraining the properties of dark energy. In: Wheeler J. C. and Martel H. (eds.) Relativistic Astrophysics Proc. 20th Texas Symposium, Melville, NY, December, 2000. AIP Conf. Proc., vol. 586, pp. 297-302. Springer, New York (2001).

25. SERENO, M.: Probing the dark energy with strong lensing by clusters of galaxies, Astron. Astrophysics 393, 757-764 (2002).

26. CHOUDHURY, T. ROY, PADMANABHAN, T.: Cosmological parameters from supernova observations: a critical 
Davis, $\mathrm{S}$.

comparison of three data sets, Astron. Astrophysics 429, 807-818 (2005).

27. IZHAK, M.: Remarks on the formulation of the cosmological constant/dark energy problems, Found. Phys. 37(10), 1470-1498 (2007).

28. DAVIS, S.: A mechanism for obtaining a rapidly decreasing cosmological term at classical scales, Research Foundation of Southern California preprint, RFSC-04-04 (2004).

29. RATRA B., PEEBles, P. J. E.: Cosmological consequences of a rolling homogeneous scalar field, Phys. Rev. D37, 3406-3427 (1988).

30. SAHNI, V, WANG, L.: A new cosmological model of quintessance and dark matter, Phys. Rev. D62, 103517:1-4 (2000).

31. ZLATEV, I., WANG, L., STEINHARDT, P. J.: Quintessance, cosmic coincidence and the cosmological constant, Phys. Rev. Lett. 82, 896-899 (1999).

32. BATCHELOR, D.: Semiclassical models for virtual antiparticle pairs, the unit of charge $e$, and the QCD coupling $\alpha_{s}$, Found. Phys. 32 (2002) 51-76. 